\title{
Diseño y validación de la Escala de Optimismo Disposicional/Pesimismo-EOP*
}

\author{
Design and Validation of the Dispositional \\ Optimism/Pesimism Scale-EOP
}

Recibido: febrero 3 de 2010 | Revisado: octubre 14 de 2010 | Aceptado: enero 24 de 2011

\author{
CONSTANZA LONDOÑo PÉREZ** \\ LinA MARÍA HERnÁNDEZ CORTÉS *** \\ IvonNe EDITH Alejo CASTAÑEDA \\ Diana Pulido GarzóN
}

Universidad Católica de Colombia, Bogotá, Colombia

\section{RESUMEN}

Este estudio tuvo como objetivo principal el diseño y validación de una escala que permitiera la evaluar la tendencia optimista/pesimista en adolescentes y jóvenes universitarios. La muestra estuvo conformada 617 estudiantes de educación superior, de ambos géneros, con una edad entre 18 y 31 años, provenientes de cuatro instituciones educativas ubicadas en dos ciudades colombianas. El análisis psicométrico mostró que la prueba cuenta con las condiciones de confiabilidad y validez necesarias para ser utilizada en poblaciones similares a la de validación. Las conclusiones muestran diferencias de género en los puntajes de optimismo y pesimismo, la presencia de sesgo optimista no realista en los adolescentes y la existencia de diferencias en la tendencia optimista según la procedencia. Se discuten las limitaciones del presente estudio.

Palabras clave autores

Optimismo, pesimismo, sesgo optimista, adolescentes.

Palabras clave descriptores

Psicometría, investigación cuantitativa, confiabilidad, validez.

Para citar este artículo: Londoño, C., Hernández, L. M., Alejo, I. E. \& Pulido, D. (2013). Diseño y validación de la Escala de Optimismo Disposicional/ Pesimismo-EOP. Universitas Psychologica, 12(1), 139-155.

* Investigación financiada por la Universidad Católica de Colombia para el diseño y validación de la Escala de Optimismo Disposicional.

** Directora Grupo de Investigación ENLACE. Facultad de Psicología, Universidad Católica de Colombia. Avenida Caracas No. 46-40 - 1er. Piso, Bogotá, Colombia.E-mail: clondono@ucatolica.edu.co

${ }^{* * * * *}$ Facultad de Psicología. E-mails: linamahernandez@gmail.com, iealejo@ucatolica.edu.co,dpulido@ucatolica.edu.co 
El optimismo, así como otros aspectos del potencial humano, ha sido parte del acervo cultural de la psicología del sentido común o psicología popular. Por ello, hasta hace muy poco, 10 años a lo sumo, han sido objeto de investigaciones científicas rigurosas que pretenden comprender este fenómeno con el nacimiento de la corriente positiva de la Psicología (Ferrando, Chico \& Tous, 2002; Vera-Villarroel, Valenzuela, Lillo, Martín \& Milos, 2008).

El optimismo disposicional y en general las creencias optimistas han sido estrechamente relacionados con mejor ajuste y afrontamiento ante diversas situaciones consideradas estresantes (Absetz, Aro, Rehnberg \& Sutton, 2000; Conway, Magai, Springer \& Jones, 2008; Chico, 2002; Gordon, Feldman, Crose \& Schoen, 2002; Grau, Hernández \& Vera-Villarroel, 2005; Kennedy \& Hughes, 2004; Martínez-Correa, Reyes del Paso, García \& González, 2006; Ouwehand, de Ridder \& Bensing, 2008; Remor, Amorós \& Carrobles, 2006; de Ridder, Schreurs \& Bensing, 2004; Waltenbaugh \& Zagummy, 2004), como puede serlo una competencia deportiva de alto rendimiento en la que la persona obtiene mejores resultados si ve como alcanzable la meta y evalúa los resultados de éxito como más probables que los negativos y triunfa, o el ingreso de los estudiantes universitarios que esperan alcanzar su meta educativa (El-Anzi, 2005; Londoño, 2009; Torres, 2004) y hasta la respuesta ante enfermedades crónicas (Carrobles, Remor \& Rodríguez-Alzamora, 2003; Conway et al., 2008; Ortiz, Ramos \& Vera-Villarroel, 2003; Segerstrom, 2006) que parece facilitar la mayor espera de resultados positivos de tratamientos complejos y por ende mejor adherencia a dichas prescripciones médicas.

El optimismo disposicional (OD) se define como un conjunto más o menos estable de expectativas positivas generalizadas acerca de la probabilidad de ocurrencia de eventos favorables (Ji, Zhang, Usborne \& Guan, 2004; Scheier \& Carver, 1985), que facilita la disposición de los recursos personales dirigidos a solucionar las situaciones estresantes. Es un afrontamiento opuesto al pesimismo, en el que la espera de eventos negativos es mayor. El OD parece ser un rasgo estable a través de la vida, aunque no hay suficiente literatura al respecto; por ahora puede decirse que tiende a ser estable en el tiempo y a través de las diferentes situaciones, y que corresponde a una cierta tendencia de las personas optimistas a explicar los eventos negativos como pasajeros y provocados por causas no permanentes y externas al individuo, en oposición al pesimista que explica los eventos negativos como derivados de una condición personal, duradera y con un efecto global (Seligman, 2003).

Para Seligman (2003), las creencias optimistas deben ser analizadas teniendo como referentes tres dimensiones: a) internalidad-externalidad: corresponde al grado en que la persona se evalúa como responsable de la ocurrencia de eventos favorables y desfavorables; b) estabilidad-inestabilidad: hace referencia a la percepción de permanencia o transitoriedad de la causa de eventos favorables y desfavorables (atribución de responsabilidad del suceso) y c) globalidad-especificidad: hace alusión al nivel de generalización o universalidad de las causas de los eventos que las hacen globales (abarcan toda las áreas de la vida de la persona) o particulares (implican una sola área o momento, es decir son circunstanciales). Los optimistas tendrán estilos explicativos con mayor externalidad, inestabilidad y especificidad frente a los eventos negativos (Remor et al., 2006) y mayor internalidad, estabilidad y globalidad frente a los eventos positivos o favorables; mientras que los pesimistas tendrían frente a los eventos desfavorables mayor internalidad, estabilidad y globalidad y frente a los eventos favorables, externalidad, inestabilidad y especificidad. Serían condiciones que se invertirían de acuerdo con el tipo de evento y la evaluación que hace el sujeto.

Así mismo, algunos autores han asociado estrechamente el OD con la eficacia personal (Gillham, Shatte, Reivich \& Seligman, 2001; Landa, Agular-Luzòn \& Salguero, 2008), pues la evaluación que hace la persona frente a la situación estresante como amenazante o no depende, en gran medida, de la visión de la capacidad personal que tiene de afrontarlo efectivamente, tal como lo habían propuesto Lazarus y Folkman (1986) y lo reconfirma Bandura (1992). Así, en el optimismo disposicional el papel de las expectativas 
generalizadas para los buenos resultados refleja la voluntad del individuo de persistir cuando se está enfrentando a la adversidad y alcanzar las metas propuestas (Londoño, 2009).

Para algunos autores no existe un tipo universal y benéfico del optimismo; puede darse un cierto tipo de optimismo no realista, a ultranza o ingenuo, que para algunos autores es equivalente a la invulnerabilidad percibida, todos referidos a una cierta tendencia a ignorar los peligros bajo la creencia de que no se está corriendo ningún riesgo: "ya que nada malo va a ocurrir y si ocurre no será a mi" (Londoño, 2007, 2009). Este tipo de pensamiento ha sido visto como poco realista ya que el individuo tiene una fuerte creencia personal de que los acontecimientos agradables tienen mayor probabilidad de ocurrirles a ellos que a otros, sumada a una menor probabilidad de eventos personales negativos (Fournier, de Ridder \& Bensing, 2002).

Estas consideraciones muestran lo complejo que resulta el fenómeno y las grandes dificultades que se pueden presentar cuando se pretende evaluar el nivel de optimismo/pesimismo que una persona tiene, ya que depende del tipo de situación, de los resultados derivados, de la evaluación que hace el sujeto y, por último, del tipo de respuesta que esta da para afrontar la situación. Se han desarrollado distintos instrumentos que intentan evaluar el optimismo como una condición de orientación vital. Ejemplo de ello es el LOT-R diseñado por Scheier, Carver y Bridges (1994) que con pocas preguntas pretende evaluar la permanencia de la visión optimista estable y generalizada de las personas. No obstante, solo está compuesto por 10 ítems de los cuales cuatro son de distracción o relleno y de los restantes, tres redactados en sentido positivo evalúan optimismo y tres redactados en sentido negativo centran su atención en la medida de pesimismo (Ferrando et al., 2002).

En diversos estudios se han analizado las propiedades psicométricas del test, obteniendo resultados que parecen demostrar su confiabilidad y validez con base en la medida de alfa de Cronbach y en el análisis de factores (Ferrando et al., 2002; Heinonen et al., 2006; Lai \& Yue, 2000; Laranjeira, 2008; Otero, Luengo, Romero, Gómez \& Castro, 1998;
Ortiz et al., 2003; Rauch, Scheweizer \& Moonsbrugger, 2008; Vera-Villarroel, Córdova-Rubio \& Celis-Atenas, 2009). No obstante, existen varias dificultades que no pueden perderse de vista. En primer lugar, el hecho de que sean solo seis ítems limita la posibilidad de validar que tan estable y permanente es el tipo de creencia positiva o negativa ante diversas situaciones de la vida, incluso si esta no corresponde más a una tendencia optimista no realista que ha sido claramente asociada a menor salud y menor probabilidad de recuperación de la salud en casos de enfermedad. En segundo lugar, un número reducido de ítems aplicados a poblaciones grandes puede llevar a un error de apreciación, ya que los índices de correlación como el alfa de Cronbach se sensibilizan demasiado (Brown, 1999) $\mathrm{y}$, tercero, los factores se ven claramente diferenciados probablemente debido a que cada uno incluye solo tres preguntas. Adicionalmente, algunos resultados acerca de la prueba son inconsistentes a través de varias culturas (Ji et al., 2004; Lai \& Yue, 2000), situación que podría explicarse tanto desde la perspectiva de las diferencias culturales como de la extensión de la prueba o la excesiva homogeneidad de las muestras (Revuelta, 2006).

Adicionalmente, se encuentran el Cuestionario de Optimismo y Pesimismo de Seligman, traducido al español por Mercé Diago y Albert Derbito (2003 citados por Seligman, 2003), que mide el nivel de optimismo de las personas. Consta de 31 ítems con dos opciones de respuesta que corresponden a situaciones en las que el evaluado elige la que más se ajusta a su forma de pensar o actuar; la distribución de los ítems en categorías permite identificar el nivel de optimismo y el de pesimismo, siendo estas las dos categorías generales analizadas. Los ítems pertenecientes a cada categoría están ordenados de forma desigual a lo largo del cuestionario. Entre las limitaciones más importantes de este instrumento se cuenta el hecho de que el nivel de confiabilidad del cuestionario original es moderado (alfa $=0.72$ ) y el de la versión traducida se desconoce, y que no se incluyen opciones de respuesta que permitan discriminar entre la existencia de un verdadero optimismo disposicional y un optimismo ingenuo no realista. 
Es por esto que es preciso desarrollar medidas confiables que faciliten la identificación de la tendencia optimista y su estabilidad en las diversas situaciones de la vida, y discriminen si este optimismo corresponde más a una visión positiva ingenua o al optimismo realista, que con altas expectativas de buen resultado disponen de sus recursos personales para hacer frente a las situaciones estresantes con la esperanza de salir adelante. Por tanto, el objetivo del presente estudio fue diseñar y validar preliminarmente una prueba de optimismo disposicional que cumpliera las características de ser relevante culturalmente, de permitir la discriminación entre optimismo disposicional y optimismo ingenuo o no realista, y cuya extensión no representara un problema, es decir, que fuera lo suficientemente extensa como para evaluar la condición optimista y lo suficientemente corta como para que no desgastara al que la respondiera.

\section{Metodología}

La muestra aleatorizada y estratificada estaba compuesta por adolescentes y jóvenes de ambos géneros, procedentes de dos ciudades colombianas, que estaban cursando alguna carrera o estudio tecnológico en instituciones de educación superior.

\section{Procedimiento}

El instrumento denominado Escala de Optimismo Disposicional/Pesimismo (EOP) que mide el nivel de optimismo disposicional/pesimismo en estudiantes universitarios, se diseño a partir de la teoria de Scheier y Carver (1985). El proceso de diseño incluyó la elaboración preliminar de 50 ítems (véase Apéndice), la evaluación de tres jurados expertos y la aplicación del instrumento para determinar sus características psicométricas a un grupo de estudiantes elegido aleatoriamente. La aplicación fue realizada por uno de los investigadores a estudiantes de diversos semestres y carreras quienes firmaron el consentimiento informado. La escala fue aplicada de forma colectiva en grupos de salones por semestres.

Las normas de calificación se basan en la puntuación de respuestas positivas de acuerdo con tres ejes opcionales: tendencia optimista, tendencia pesimista y tendencia al sesgo optimista no realista, dados por tres opciones de respuesta ofrecidas para cada ítem y la elección hecha por quien contestaba el cuestionario.

\section{Resultados}

La muestra aleatorizada y estratificada estaba compuesta por 617 adolescentes y jóvenes, 294 hombres (48 \%) y 323 mujeres (52\%), procedentes de dos ciudades colombianas (Ibagué y Bogotá) que estaban cursando alguna carrera o estudio tecnológico en cuatro instituciones de Educación Superior ubicadas en dichas ciudades, cuyo tamaño correspondía al $10 \%$ del total de matriculados a la fecha de aplicación. Las edades oscilaban entre los 16 y los 32 años con una media de 19.2 años, es decir que la muestra incluyó tanto adolescentes como jóvenes (Tabla 1).

\section{Análisis de las condiciones psicométricas de la prueba}

Los conceptos emitidos por los expertos mostraron un alto nivel de concordancia entre ellos (89\%) respecto de la importancia de tener en cuenta las situaciones definidas en los ítems y acerca de la

TABLA 1

Descripción de la muestra

\begin{tabular}{|c|c|c|c|c|c|}
\hline Variable & $N$ & $\%$ & Procedencia & $N$ & $\%$ \\
\hline Hombre & 294 & 48 & Ibagué & 245 & 40 \\
\hline \multirow[t]{2}{*}{ Mujer } & 323 & 52 & Bogotá & 362 & 60 \\
\hline & $\mathbf{M}$ & $\mathrm{DE}$ & Rango & & \\
\hline Edad & 19.2 & 5.53 & 16 a 32 & & \\
\hline
\end{tabular}

Fuente: elaboración propia. 
pertinencia de las opciones de respuesta ofrecidas para evaluar la tendencia optimista, pesimista o la existencia de sesgo optimista no realista. Respecto de la redacción, los evaluadores sugirieron unificar la extensión de los ítems y variar la redacción de las preguntas 8 y 14. Una vez realizados dichos ajustes se hizo una aplicación previa a la piloto para determinar las condiciones de validez de presentación del instrumento y se evidenció que no había problemas en la comprensión de las preguntas ni en la elección de las opciones de respuesta.

De acuerdo con los análisis psicométricos adelantados con los 50 ítems, la prueba contaba con un nivel alto de confiabilidad $(\alpha=0.085)$, que se conservó al elegir los 22 mejores ítems, ya que se buscaba obtener una versión corta que facilitara su uso posterior en la población (Apéndice A).

El análisis por ítem de las 50 preguntas mostró que todas aportaban en la misma medida a la confiabilidad general de la prueba, por ello se examinó el comportamiento de elección de los participantes frente a las opciones de respuesta y se seleccionaron los 22 ítems en los que las respuestas dadas por el total de los participantes estaban equilibradas respecto de las opciones ofrecidas (Tabla 2).

\section{Calificación}

Se normalizaron los puntajes y se identificaron los puntos de corte de cada tendencia. Para obtener la calificación se dieron dos puntos en cada tendencia (Op: optimismo; Ps: pesimismo; SONR: sesgo optimista no realista) por cada una de las respuestas, de acuerdo con la clave de puntuación. Para sacar conclusiones acerca de las tendencias se analizan las calificaciones en cada una: la primacía de un estilo se ve reflejada en diferencias mayores a tres puntos
TABLA 2

Análisis de la confiabilidad por ítems

\begin{tabular}{ccc}
\hline ítem & $\begin{array}{c}\text { Correlación con } \\
\text { la escala total }\end{array}$ & $\begin{array}{c}\text { alfa de Cronbach } \\
\text { item borrado }\end{array}$ \\
\hline p6 & 0.267 & 0.805 \\
p7 & 0.292 & 0.804 \\
p15 & 0.423 & 0.797 \\
p17 & 0.28 & 0.807 \\
p23 & 0.442 & 0.796 \\
p24 & 0.352 & 0.801 \\
p25 & 0.396 & 0.799 \\
p31 & 0.361 & 0.8 \\
p32 & 0.322 & 0.802 \\
p33 & 0.345 & 0.802 \\
p37 & 0.345 & 0.801 \\
p40 & 0.321 & 0.802 \\
p4 & 0.308 & 0.803 \\
p9 & 0.332 & 0.802 \\
p14 & 0.343 & 0.801 \\
p21 & 0.31 & 0.803 \\
p22 & 0.496 & 0.795 \\
p35 & 0.386 & 0.799 \\
p39 & 0.506 & 0.794 \\
p41 & 0.524 & 0.793 \\
p43 & 0.374 & 0.8 \\
p47 & 0.341 & 0.801 \\
\hline
\end{tabular}

Fuente: elaboración propia.

entre los puntajes obtenidos. La acumulación mayor de respuestas en una de las tendencias denota la existencia de un estilo particular de evaluar y afrontar las situaciones.

\section{Optimismo}

Al analizar las tendencias en la población estudiada se observó en las mujeres una media de optimismo significativamente mayor que la de los hombres, y

TABLA 3

Descripción de la población

\begin{tabular}{lcccccccc}
\hline & Mujeres & \multicolumn{3}{c}{ Hombres } & \multicolumn{3}{c}{ Ibagué } & \multicolumn{3}{c}{ Bogotá } \\
\cline { 2 - 9 } & $\mathbf{M}$ & $\mathrm{DE}$ & $\mathbf{M}$ & $\mathrm{DE}$ & $\mathbf{M}$ & $\mathrm{DE}$ & $\mathrm{M}$ & $\mathrm{DE}$ \\
\hline Optimismo & 16.73 & 4.21 & 13.82 & 4.12 & 14.34 & 3.02 & 17.23 & 4.52 \\
Sesgo opt. & 4.09 & 0.83 & 4 & 2.01 & 4.22 & 2.25 & 4.19 & 0.86 \\
Pesimismo & 3.46 & 1.11 & 6.41 & 2.97 & 6.23 & 2.95 & 2.17 & 1.35 \\
\hline
\end{tabular}

Fuente: elaboración propia. 
una significativamente menor en pesimismo, sin diferencias importantes en el sesgo optimista no realista. Respecto de la procedencia, se evidenció que los bogotanos obtuvieron una media de optimismo significativamente más alta que la obtenida por los ibaguereños, sin diferencias significativas en las tendencias pesimistas y de sesgo optimista no realista (Tabla 3).

\section{Discusión y conclusiones}

Al realizar el análisis psicométrico se concluye que el instrumento alcanzó un nivel moderado alto de confiabilidad y puede ser usado para discriminar la tendencia a ser optimista, pesimista o el sesgo optimista no realista, en población entre 16 a 35 años. Incluso, teniendo en cuenta el tamaño de la muestra y la aleatorización, es posible que su uso sea extendido a jóvenes incluidos en programas de educación superior colombianos. No obstante, debe diversificarse aún más la muestra cuidando incluir jóvenes de diversas regiones de país, para poder adelantar estudios culturales comparativos. Adicionalmente, al revisar las situaciones descritas en los ítems, se evidenció la tendencia a describir principalmente eventos cotidianos; por ello, para aumentar la capacidad discriminativa de la prueba, sería deseable incluir en las preguntas otras situaciones que representen mayor estrés para el individuo y así evaluar si aún persiste la visión optimista. Igualmente, se recomienda ajustar la forma de calificar el cuestionario para que las tendencias se hagan más evidentes en el perfil final.

\section{Optimismo-pesimismo}

Respecto del optimismo se evidenciaron diferencias de género, al igual que en el estudio conducido por Londoño (2009) en una población similar y los realizados por otros investigadores en distintas poblaciones (Casullo \& Castro, 2000; Elgar, Arlett \& Groves, 2003; Landis et al., 2007; Peterson, 2000). Las mujeres mostraron una tendencia mayor que los hombres hacia el optimismo disposicional y los hombres una marcada tendencia hacia el pesimismo. Aunque en ninguno de los estudios se planteó como interrogante la explicación de esta diferencia por género, sí resultan llamativos estos hallazgos y es recomendable que este sea un tema posterior de investigación.

En la misma línea, el presente estudio y el de Londoño (2009) mostraron la existencia de diferencias entre quienes viven en Bogotá y quienes viven en otras ciudades. Sin pretender dar explicaciones de causalidad que trasciende del todo los alcances del presente estudio, podría pensarse que las personas de Bogotá perciben la existencia de mayores oportunidades culturales, sociales, educativas y laborales que facilitaran el alcance de los objetivos de vida a largo plazo, comparativamente con las ubicadas en ciudades intermedias que no tienen la mismas oportunidades, e incluso tienden a pensar de forma pesimista que la probabilidad de alcanzar las metas propuestas es baja.

Ya Contreras y Esguerra (2006) evidenciaron la relación entre el optimismo y las expectativas hacia el futuro, específicamente en el aumento de la sensación de control y de dar sentido a las experiencias vividas. En la misma línea de Londoño (2007), Covey y Davies (2004), Carr (2007) y Seligman (2003), se encontró que los adolescentes tienen una visión optimista no realista acerca de los eventos y sus consecuencias, y que con el pasar del tiempo y la ocurrencia de eventos estresantes los jóvenes reducen el optimismo a ultranza haciéndose más realistas.

Finalmente, es importante reconocer las limitaciones del presente estudio en la obtención de una muestra procedente de diversas ciudades, debido a la falta de redes de investigación efectivas que faciliten la consecución de muestras más universales y así poder afrontar la estandarización y baremación de la escala. De otra parte, más que una limitación, se debe resaltar la necesidad de conocer cuáles factores facilitan la adquisición de un estilo optimista realista o funcional que favorezca el bienestar de ser optimista.

\section{Referencias}

Absetz, P., Aro, A., Rehnberg, G. \& Sutton, S. (2000). Comparative optimism in the opinion of the risk 
of the chest cancer: Effects of the knowledge of factor of the experience and risk. Psychology Health \& Medecine, 5(4), 367-391.

Bandura, A. (1992). A self-efficay mechanism in psychobiologic functioning. En R. Schwarzer (Ed.), Selfefficacy: Thought control of action (pp. 3-38). Washington, DC: Hemisphere Publishing Corp.

Brown, G. F. (1999). Principios de la medición en psicología y educación. México: Manual Moderno.

Carr, A. (2007). Psicología positiva: la ciencia de la felicidad. Barcelona: Paidós.

Carrobles, J., Remor, E. \& Rodríguez-Alzamora, L. (2003). Afrontamiento, apoyo social percibido y distrés emocional en pacientes con infección por VIH. Psicothema, 15(3), 420-426.

Casullo, M. \& Castro, A. (2000). Evaluación del bienestar psicológico en estudiantes adolescentes argentinos. Revista de Psicología. Pontificia Universidad Católica del Perú, XVIII(1),35-68.

Chico, E. (2002). Optimismo disposicional como predictor de estrategias de afrontamiento. Psicothema, 14(3), 544-550. Recuperado el 12 de septiembre de 2006, de http://dialnet.unirioja.es/servlet/articulo? codigo $=1103085 \&$ \&orden $=33104 \&$ info $=$ link

Contreras, F. \& Esguerra, G. (2006). Psicología positiva: una nueva perspectiva en psicología. Diversitas, 2 (2), 311-319.

Conway, F., Magai, C., Springer, C. \& Jones, S. (2008). Optimism and pessimism as predictors of adjustment. Journal of Research in Personality, 42(5), 1352-1357.

Covey, J. \& Davies, A. (2004). iAre people little realistic optimist? She depends how you ask to him. British Journal of Health Psychology, 9(1) 39-50.

El-Anzi, F. O. (2005). Academia achievement and its relationship with anxiety, self-esteem, optimism, and pessimism in Kuwaiti students. Social Behavior and Personality, 33(1), 95-103.

Elgar, F., Arlett, C. \& Groves, R. (2003). Stress, coping, and behavioural problems among rural and urban adolescents. Journal of Adolescence, 26(5), 574-585.

Ferrando, P., Chico, E. \& Tous, J. (2002). Propiedades psicométricas del test de optimismo Life Orientation Test. Psicothema, 14(3), 673-680.

Fournier, M., de Ridder, D. \& Bensing, J. (2002). Optimism and adaptation to chronic disease: The role of optimism in relation to self-care options of type I diabetes mellitus, rheumatoide arthritis and multiple sclerosis. British Journal of Health Psychology, 7(4), 409-432.

Gillham, J. E., Shatté, A. J., Reivich, K. J. \& Seligman, M. E. P. (2001). Optimism, pessimism, and explanatory style. En E. C. Chang (Ed.), Optimism \& pessimism (pp. 53-75). Washington, DC: American Psychological Association.

Gordon, A., Feldman, D., Crose, R. \& Schoen, E. (2002). The paper of the religious belief in facing chronic disease. Counseling and Values, 46(3), 162-174.

Grau, J., Hernández, E. \& Vera-Villarroel, P. (2005). Estrés, salutogénesis y vulnerabilidad. En E. Hernández \& J. Grau (Eds.), Psicología de la salud: fundamentos, metodología, aplicaciones (pp. 113-177). México: Universidad de Guadalajara.

Heinonen, K., Raikkonen, K., Matthews, K. A., Scheier, M. F., Raitakari, O. T., Pulkki, L., et al. (2006). Socioeconomic status in childhood and adulthood: Associations with dispositional optimism and pessimism over a 21-year follow-up. Journal of Personality, 74(4), 1111-1126.

Ji, L., Zhang, Z., Usborne, E. \& Guan, Y. (2004). Optimism across cultures: In response to the severe acute respiratory syndrome outbreak. Asian Journal of Social Psychology, 7(1), 25-34.

Kennedy, D. K. \& Hughes, B. M. (2004). The optimismneuroticism question: An evaluation based on cardiovascular reactivity in female college students. The Psychological Record, 54(3), 373-386.

Lai, L. \& Yue, X. (2000). Measuring optimism in Hong Kong and mainland Chinese with the revised Life Orientation Test. Personality and Individual Differences, 28(4), 781-796.

Landa, A., Agular-Luzòn, M. C. \& Salguero, M. F. (2008). El papel de la IEP y del optimismo/pesimismo disposicional en la resolución de problemas sociales. Revista Electrónica de Investigación Psicoeducativa, 15(6), 363-382.

Landis, D., Gaylord-Harden, N., Malinowski, S., Grant, K., Carleton, R. \& Ford, R. (2007). Urban adolescent stress and hopelessness. Journal of Adolescence, 30(6), 1051-1070. 
Laranjeira, C. A. (2008). Tradução e validação portuguesa do revised Life Orientation Test (LOT-R). Universitas Psychologica, 7(2), 469-476.

Lazarus, R. \& Folkman, S. (1986). Estrés y procesos cognitivos. Barcelona: Martínez Roca.

Londoño, C. (2007). Construcción de un modelo cognitivo social integrado para la prevención del abuso de alcohol en universitarios bogotanos. Tesis de Maestría, Facultad de Psicología, Universidad Nacional de Colombia, Bogotá.

Londoño, C. (2009). Optimismo y salud positiva como predictores de la adaptación a la vida universitaria. Acta Colombiana de Psicología, 12(1), 95-107.

Martínez-Correa, A., Reyes del Paso, G., García-León, A. \& González-Jareño, M. (2006). Optimismo/ pesimismo disposicional y estrategias de afrontamiento del estrés. Psicothema, 18(1), 66-77. Recuperado el 4 de septiembre de 2006, de http://www. psicothema.com/psicothema.asp? ID $=3177$

Ortiz, J., Ramos, N. \& Vera-Villarroel, P. E. (2003). Optimismo y salud: estado actual e implicaciones para la Psicología Clínica y de la Salud. Suma Psicológica, 10(1), 119-134.

Otero, J. M., Luengo, A., Romero, E., Gómez, J. A. \& Castro, C. (1998). Psicología de la personalidad. Manual de prácticas. Barcelona: Ariel Practicum.

Ouwehand, C., de Ridder, D. \& Besing, S. (2008). Effects of temptation and weight on hedonics and motivation to eat in women. Obesity, 16(8), 1788-1793.

Peterson, C. (2000). The future of optimism. American Psychologist, 55(1), 44-55.

Rauch, W. A., Schweizer, K., \& Moosbrugger, H. (2008). An IRT analysis of the Personal Optimism Scale. European Journal of Psychological Assessment, 24(1), 49-56.

Remor, R., Amorós, M. \& Carboles, J. (2006). El optimismo y la experiencia de ira en relación con el malestar físico. Anales de Psicología, 22(1), 37-44.

Recuperado el 11 de octubre de 2006, de http:// redalyc.uaemex.mx/redalyc/src/inicio/ArtPdfRed. jsp?iCve $=16722105 \&$ iCveNum $=0$

Ridder de, D., Shreurs, K. \& Bensing, J. (2004). The relative benefits of being optimistic: Optimism as a coping resource in multiple sclerosis and Parkinson disease. British Journal of Health Psychology, 5(2), 141-155.

Scheier, M. \& Carver, C. (1985). Optimism, coping and health: Assessment and implications of generalized outcome expectancies. Health Psychology, 4(3), 219-247.

Scheier, M., Carver, C. \& Bridges, M. (1994). Distinguishing optimism from neuroticism (and trait anxiety, self-mastery, and self-esteem): A reevaluation of the Life Orientation Test. Journal of Personality and Social Psychology, 67(6), 1063-1078.

Segerstrom, S. (2006). How does optimism suppress immunity? Evaluation of three affective pathways. Health Psychology, 25(5), 653-657.

Seligman, M. E. (2003). La auténtica felicidad. Barcelona: Vergara.

Torres, K. (2004). Reveille for radicals! The paramount purpose of health education? Health Education Research, 17(1), 1-5.

Vera-Villarroel, P., Córdova-Rubio, N. \& Celis-Atenas, K. (2009). Evaluación del optimismo: un análisis preliminar del Life Orientation Test versión revisada (LOT-R) en población chilena. Universitas Psychologica, 8(1), 61-68.

Vera-Villarroel, P., Valenzuela, P., Lillo, S., Martín, N. \& Milos, I. (2008). Felicidad y optimismo: estado actual de la investigación. Manuscrito enviado para publicación.

Waltenbaugh, A. \& Zagummy, M. (2004). Diagonal optimism and control percibe between. Journal of Alcohol and Drug Education, 47(3), 20-34. 


\title{
Apéndice
}

\section{UNIVERSIDAD CATÓLICA DE COLOMBIA FACULTAD DE PSICOLOGÍA}

\author{
Cuestionario Optimismo Disposicional \\ JÓVENES Y ADOLESCENTES
}

El presente cuestionario pretende identificar la forma como usted actúa o piensa en diversas situaciones de la vida diaria; ya que algunas situaciones son hipotéticas, por favor responda teniendo en cuenta sus pensamientos y acciones más habituales en situaciones similares.

\section{Me invitan a una fiesta y no encuentro la dirección del sitio}

a) Pienso que es posible que haya anotado mal la dirección y llamo, porque sé que pronto estaré pasándola bien.

b) Después de 1 hora sigo buscando porque sé que tarde o temprano la encontraré.

c) Busco 5 minutos y pienso que definitivamente no la voy a encontrar y que lo mejor es devolverme.

\section{Tengo una pelea con mi pareja}

a) Es preciso que hablemos para saber que le ha molestado y buscar soluciones porque todo puede mejorar.

b) No importa lo que pase nuestro amor es eterno y los defectos los superaremos de cualquier manera.

c) Esto es espantoso, todo se acabó y nada puedo hacer.

\section{Incumplo una cita importante con mi jefe y debo llamarlo para pedirle un permiso}

a) Es posible que me diga que no porque he fallado, intentaré suplir la falla y organizar mejor mi tiempo.

b) No importa lo que pase, con seguridad obtendré el permiso.

c) No llamaré porque de seguro está enfadado y me va a despedir.

\section{Me inscribo para concursar por una beca y pierdo}

a) Había muchas personas inscritas, habrá una segunda oportunidad.

b) Esperaré, de pronto haya alguien que la rechace.

c) Eso se sabía, siempre hay alguien mejor que yo.

\section{Un amigo me pide ayuda para realizar un trabajo}

a) Le parezco muy inteligente y por eso confía en mí.

b) Veré en que le puedo ayudar, seguro se me ocurrirá algo.

c) Pobrecito, si supiera... yo no soy capaz ni con mi propio trabajo.

\section{Cuando realizo trabajos en grupo}

a) Se presentan discusiones interesantes y grandes aportes.

b) Seguro que me va bien ya que varias cabezas piensan más que una.

c) Mi opinión no cuenta, así que el trabajo lo van a hacer ellos. 


\section{Guardo una copia cuando realizo un trabajo}

a) Es mejor prevenir que lamentar.

b) Pienso que no es necesario, nada va a pasar.

c) Soy tan de malas que seguro se me borra.

\section{Me felicitan por mi buen desempeño como estudiante}
a) Me esforcé bastante tratando de hacer lo mejor.
b) Siempre me va bien, sin importar lo que haga.
c) No lo hubiera podido hacer solo.

\section{Tenía guardado un trabajo importante y no lo pude imprimir}
a) A lo mejor no lo guarde correctamente.
b) Seguiré intentado, es imposible que se dañe.
c) A mí siempre me pasa... y me sigue pasando.

\section{Presento un examen y me llaman urgentemente}

a) Se me olvidaría contestar una pregunta.

b) Me necesitan para felicitarme.

c) Seguro que lo perdí.

11. Preparé por primera vez una comida y los invitados apenas probaron bocado

a) El ambiente de la cena talvez no estaba muy alegre.

b) Ya habían comido antes y por eso probaron tan poco.

c) Mi comida estaba tan fea que prefirieron hacerme el desplante.

\section{Me varé por gasolina en un lugar oscuro y solo}

a) Espero que pase otro conductor que me pueda regalar gasolina.

b) Empujaré el carro hasta la estación más cercana.

c) Por no tener cuidado ahora me pueden atracar.

\section{Me solicitan unos exámenes médicos}

a) Son exámenes necesarios así que me los realizaré.

b) No necesito hacerme exámenes porque me siento bien.

c) Me da miedo porque sé que me van salir mal.

\section{Me proponen tener relaciones sexuales}

a) Si utilizo un buen método anticonceptivo probablemente no habrá problemas.

b) Con una sola vez seguro no tendré problemas.

c) Si lo hago, seguramente se me pegará alguna enfermedad.

15. Me tomé unos tragos e hice el ridículo delante de mis amigos

a) Les pido disculpas y pienso que debo controlar mejor estas situaciones.

b) A nadie le molesta lo que los borrachos hacen, pronto lo olvidarán.

c) Ahora piensan que soy lo peor y no me volverán a invitar a salir. 
16. Un amigo me causa un disgusto muy grande

a) Espero a calmarme, antes de hablar con él.

b) Entre amigos todo se perdona.

c) Esta amistad se acaba para siempre.

17. Con frecuencia me invitan a jugar play station

a) Sé jugar muy bien, además soy muy divertido.

b) Les gusta perder, pues soy invencible.

c) Soy el único desocupado que tiene tiempo de jugar con ellos.

18. Digo una broma y nadie se ríe

a) Seguramente no me entendieron.

b) No soy muy gracioso para hacer bromas.

c) Me veo mejor callado.

19. Se me olvidó pagar el recibo de la luz

a) Seguro en el CADE me solucionan el inconveniente.

b) Es imposible que me corten la luz.

c) Lo más seguro es que mañana la corten.

\section{Se me perdieron todos mis documentos}

a) Coloco un aviso en los lugares en donde estuve para recuperarlos.

b) Creo que me llamarán pronto y me los devolverán.

c) Me da miedo que quien los encuentre los utilice con malas intenciones.

21. En un almacén le dan bonos de descuento a todos menos a mí

a) Probablemente en otra oportunidad me ganaré algo.

b) Con seguridad me ganaré un premio muchísimo mejor.

c) Hasta en esas ocasiones las personas me rechazan.

22. Mi pareja me hace un regalo poco usual y sin razón aparente

a) Es detallista y me quiere mucho.

b) Lo tengo bajo mi dominio.

c) Me está poniendo los cachos y se está sintiendo culpable.

23. Mi médico me dice que consumo demasiada grasa

a) El cuidado de mi salud depende de mi alimentación.

b) Hay gente que sin cuidarse no se enferma.

c) De pronto tengo algo grave por no cuidar mi alimentación.

24. Me caigo con frecuencia mientras practico algún deporte

a) Tengo que practicar para mejorar la técnica.

b) Soy realmente bueno y quieren lesionarme.

c) Definitivamente no sirvo para esto. 


\section{Me entregan una mala calificación en una materia}

a) Si estudio un poco más voy a tener éxito.

b) La próxima será mejor como sea sacaré buena nota.

c) No sirvo para estudiar y por eso me va mal.

26. El bus que esperaba se me pasó y voy a llegar tarde

a) Debo llamar y avisar que me retrasaré en la hora de llegada.

b) El siguiente bus me llevará muy rápido.

c) Me voy a meter en un problema grave por mi tardanza.

27. Me doy cuenta que mis vecinos saludan a todos menos a mí

a) No los conozco y por eso no me saludan.

b) Me tienen envidia.

c) A todo el mundo le caigo mal.

28. Debo hacer un trabajo y se me olvida conseguir algo importante para hacerlo

a) Aunque me retrase debo comprar lo que me falta.

b) Voy a poder hacerlo bien a pesar de que no tenga todo el material.

c) Es imposible que me acuerde de todo.

\section{Voy a presentar mi nuevo novio a mi familia}

a) En casa son comprensivos y van aprobar mi elección.

b) Les va a caer superbien a todos.

c) Lo más seguro es que no les guste y lo van a criticar.

\section{Estoy saliendo con alguien y hace un mes no me llama}

a) Quizá no le interesaba lo suficiente.

b) No ha podio llamarme, pero sé que le hago falta.

c) De pronto le ocurrió algo muy grave y no me ha podido llamar.

\section{Presento una sugerencia a mi grupo y no me prestan atención}

a) No me escucharon, volveré a expresar mi idea.

b) Están distraídos y no se fijaron en mi comentario.

c) Digo cosas tan malas que no tienen ninguna importancia.

32. Constantemente las personas me dicen que tengo apariencia de estar enfermo(a)

a) Aunque no me alarmo demasiado, pienso en hacerme un chequeo.

b) Nunca me enfermo, no sé que les pasa.

c) Estoy realmente enfermo(a) y no me había dado cuenta.

\section{Timbra el teléfono a media noche}

a) Voy a contestar para ver qué ocurre.

b) Qué bueno que alguien piense en mí a esta hora de la noche.

c) Pasó algo malo, a alguien le ocurrió un accidente. 


\section{Un miembro de mi familia no llega a la hora usual a casa}

a) Busco comunicarme para saber a que hora llega.

b) Se quedaría bailando o con un conocido, pronto llegará.

c) Pasó algo malo, posiblemente tubo un accidente.

\section{Se me viene la sangre por la nariz}

a) Seguro fue por el cambio de clima, descansaré un rato y pronto pasará.

b) No importa lo que haga, tarde o temprano dejaré de sangrar.

c) Esto es el indicio de una grave enfermedad que los médicos no me han detectado.

36. Veo que una persona cae inconsciente en la calle, parece ser grave y se necesita a un médico

a) Subo a la persona a un carro para llevarla al hospital más cercano y salvar su vida.

b) Empiezo a gritar "un médico" y estoy convencido de que entre las personas que observan hay uno.

c) Rompo en llanto, pues estoy seguro que no hay nada que hacer por la persona, ha muerto.

37. El colectivo en el que me trasportaba se varó

a) Pregunto si la reparación no demorará, de lo contrario pido mi dinero y tomo otro transporte.

b) Espero porque estoy seguro de que la reparación no demorará.

c) Pienso que lo único que falta es que también haya trancón y llegue mucho más tarde.

\section{Me llaman para una entrevista de trabajo}

a) Pienso que tengo posibilidad de acceder al trabajo, me preparo y voy con las mejores expectativas.

b) Creo que no es necesario prepararme mucho, pues si me llamaron es seguro que el puesto es mío.

c) Pienso en no ir, pues es seguro que no tengo posibilidad frente a los otros aspirantes al puesto.

\section{Me han puesto una sanción por no pagar la multa de la biblioteca}

a) Pago la multa y espero que el período de sanción no sea muy largo.

b) No pago la multa y estoy seguro que si insisto al bibliotecario obtendré el préstamo.

c) Para que pagar si igual me van a sancionar.

40. Un amigo me pide que compre una boleta de una rifa

a) Compro la rifa por ayudar a mi amigo, pero sé cuál es mi verdadera posibilidad ganar.

b) Compro la rifa porque pienso que tengo muy buena suerte y me ganaré el premio.

c) Compro la rifa para que mi amigo no piense que soy tacaño, aunque soy de mala suerte.

\section{Debo exponer sobre un tema que no manejo}

a) Investigo, acudo a varias fuentes y me preparo porque la idea es aprender.

b) Investigo en un solo libro porque creo que es suficiente.

c) Hago una presentación muy elaborada y repaso hasta el final, aunque sé que me va a ir mal.

\section{Mi jefe o amigo me pide un consejo}

a) Opino sobre la situación dejando en claro que es solo un punto de vista.

b) Opino sobre la situación seguro de que tengo toda la razón.

c) Prefiero no opinar porque seguro resulto metido en problemas. 
43. Salí distraído de mi casa y olvidé que tenía pico y placa

a) Busco un sitio seguro donde estacionarme y espero a que pase el pico y placa.

b) Continúo mi trayecto pues estoy convencido de que pasaré desapercibido y no me multarán.

c) Entro en desesperanza, no encontraré un lugar donde estacionarme y me van a sancionar.

\section{Me llaman a cobrarme una deuda}

a) Pido un plazo razonable en el que pueda conseguir el dinero.

b) Digo que al día siguiente pagaré y pienso que será fácil conseguirlo.

c) Pienso que es imposible conseguir el dinero y que inevitablemente quedaré mal.

\section{Olvidé el cumpleaños de mi pareja}

a) Me excuso y asumo mi responsabilidad, proponiendo una celebración.

b) Creo que un buen regalo cura cualquier incomodidad sin importar la falla.

c) Pienso que esta puede ser la disculpa para deshacerse de mí.

46. Me ofrecen la oportunidad de irme a estudiar al exterior

a) Analizo la viabilidad de la oferta y los beneficios antes de tomar cualquier decisión.

b) No lo pienso dos veces, lo importante es aprovechar la oportunidad pase lo que pase.

c) Siento temor de las cosas malas que puedan ocurrir y prefiero rechazar la oportunidad.

47. Un amigo me propone que montemos un negocio de comidas rápidas

a) Analizo la viabilidad del negocio y los beneficios antes de tomar cualquier decisión.

b) Invierto con la certeza de que tendré buenas ganancias muy pronto.

c) Busco una excusa porque sé que el negocio fracasará y además perderé un amigo.

48. El conductor del colectivo me dice que más tarde me da las vueltas

a) Creo alguna estrategia que me permita recordar que debo pedir las vueltas antes de bajarme.

b) Me siento y confío en que el señor del colectivo se acordará de entregarme las vueltas.

c) Solicito cada 5 minutos mis vueltas pues estoy seguro de que si no lo hago se me olvidará el dinero.

\section{He aumentado de peso}

a) Pienso que debo programar una dieta y creo que puedo cumplirla.

b) Pienso que un cualquier momento adelgazaré, no hay que preocuparse demasiado.

c) Me lamento porque es imposible bajar de peso, nada de lo que haga dará resultado.

\section{Me han negado la solicitud de crédito educativo para estudiar en la universidad}

a) Busco otras alternativas de crédito que se ajusten a mis posibilidades.

b) Insisto porque la negación pudo deberse a un error de ellos.

c) Creo que me rechazaron porque no podré pagar la deuda. 


\section{Cuestionario Optimismo Disposicional (ADULTOS)}

Código de aplicación:

El presente cuestionario pretende identificar la forma como usted actúa o piensa en diversas situaciones de la vida diaria; ya que algunas situaciones son imaginadas, por favor responda teniendo en cuenta lo que piensa y hace comúnmente, subraye la frase elegida.

1. Me invitan a una fiesta y no encuentro la dirección

a) Llamo para confirmar la dirección, sé que pronto estaré pasándola bien.

b) Después de 1 hora sigo buscando, sé que tarde o temprano la encontraré.

c) Busco solo 5 minutos, sé que no la voy a encontrar y me devuelvo para la casa.

\section{Un amigo me pide ayuda para resolver un problema}

a) Le parezco muy inteligente y por eso confía en mí.

b) Veré en que le puedo ayudar, seguro se me ocurrirá algo.

c) Si supiera que no soy capaz ni con mis problemas,

\section{Me felicitan por mi buen desempeño como estudiante}

a) Me esforcé bastante tratando de hacer lo mejor.

b) Siempre me va bien, sin importar lo que haga.

c) No lo hubiera podido hacer solo.

\section{Invité a unos amigos a una reunión en mi casa y la mayoría rechazó la comida}

a) No había buen ambiente en la fiesta.

b) Ya habían comido antes y por eso comieron poco.

c) La comida estaba tan fea que prefirieron decir que no.

\section{Me solicitan unos exámenes médicos}

a) Son exámenes necesarios así que me los realizaré.

b) No necesito hacerme exámenes porque me siento bien.

c) Me da miedo porque sé que me van salir mal.

6. Me tomé unos tragos e hice el ridículo delante de mis amigos

a) Les pido disculpas y pienso que debo controlar mejor estas situaciones.

b) A nadie le molesta lo que los borrachos hacen, pronto lo olvidarán.

c) Ahora piensan que soy lo peor y no me volverán a invitar.

\section{Un amigo me saca la piedra}

a) Espero a calmarme antes de hablar con él.

b) Entre amigos todo se perdona.

c) Esta amistad se acaba para siempre.

\section{Hecho un chiste y nadie se ríe.}

a) Seguramente no lo entendieron.

b) No soy muy bueno para hacer bromas.

c) Me veo mejor callado. 


\section{Se me perdieron todos mis documentos}

a) Sé que los encontraré por eso pongo un aviso en donde estuve para recuperarlos.

b) Creo que me llamarán pronto y me los devolverán.

c) Me da miedo que quien los encuentre los utilice con malas intenciones.

10. En un almacén le regalan bonos de descuento a todos, menos a mí

a) Probablemente en otra oportunidad me ganaré algo.

b) Con seguridad me ganaré un premio muchísimo mejor.

c) Cuando me vaya fijo traen más y le dan a todo el mundo.

11. Mi pareja me da un regalo sin razón aparente

a) Es detallista y me quiere mucho.

b) Lo tengo bajo mi dominio.

c) Me puso los cachos y se siente culpable.

12. Mi médico me dice que como mucha grasa

a) Puedo mejorar mi salud si como mejor.

b) Hay gente que no se cuida y no pasa nada.

c) Si estoy gordo no hay nada que hacer.

\section{Me equivoco con frecuencia en mi trabajo}

a) Tengo que practicar para mejorar.

b) Soy realmente bueno pero las cosas no se me dan.

c) Nada que hacer, voy a perder mi trabajo.

\section{El bus que esperaba se me pasó y voy a llegar tarde}

a) Debo llamar y avisar que voy tarde.

b) El siguiente bus me llevará muy rápido.

c) Me voy a meter en un problema grave por mi tardanza.

\section{Timbra el teléfono a media noche}

a) Voy a contestar para ver qué ocurre.

b) Qué bueno que alguien piense en mí a esta hora de la noche.

c) Pasó algo malo, a alguien le ocurrió un accidente.

\section{Un miembro de mi familia no llega a la hora usual a casa}

a) Busco comunicarme para saber a qué hora llega.

b) Se quedaría bailando o con un conocido, pronto llegará.

c) Pasó algo malo, posiblemente tubo un accidente.

17. El colectivo en el que me trasportaba se varó

a) Pregunto si la reparación no demorará de lo contrario pido mi dinero y tomo otro transporte.

b) Espero porque estoy seguro de que la reparación no demorará.

c) Pienso que lo único que falta es que también haya trancón y llegue mucho más tarde. 
18. Me llaman para una entrevista de trabajo

a) Pienso que tengo posibilidad de acceder al trabajo, me preparo y voy con las mejores expectativas.

b) Creo que no es necesario prepararme mucho, pues si me llamaron es seguro que el puesto es mío.

c) Pienso en no ir, pues es seguro que no tengo posibilidad frente a los otros aspirantes al puesto.

19. Me llaman a cobrarme una deuda

a) Pido un plazo razonable en el que pueda conseguir el dinero.

b) Digo que al día siguiente pagaré y pienso que será fácil conseguirlo.

c) Pienso que es imposible conseguir el dinero y que inevitablemente quedaré mal.

\section{Olvidé el cumpleaños de mi pareja}

a) Me excuso y asumo mi responsabilidad, proponiendo una celebración.

b) Creo que un buen regalo cura cualquier incomodidad sin importar la falla.

c) Pienso que esta puede ser la disculpa para deshacerse de mí. 
